DOI: http://dx.doi.org/10.14201/0AQ02874154

\title{
A DEEP TECH ARCHITECTURE FOR INTELLIGENT IOT SYSTEMS
}

\section{Mehmet OzTurk}

BISITE Digital Innovation Hub, University of Salamanca, Edificio Multiusos $I+D+I, 37007$, Salamanca, Spain

mehmet@usal.es

Aвstract: The increase in the number of connected devices on the Internet of Things (IoT), interactions and the amount of data raises a number of issues. Two major problems are limitations in terms of network latency and bandwidth. While cloud-based infrastructures give us access to scalable, on-demand storage and processing services that can scale to the requirements of the Internet of Things (IoT), these centralized resources can create unacceptable delays and performance problems for devices that have latency-sensitive applications, such as health monitoring and emergency response applications. This article has been created for the PhD thesis that aims to create a deep tech architecture for intelligent IoT systems.

Keywords: Edge Computing; Machine Learning; Deep Tech; Internet of Things (IoT).

\section{Introducción}

The recent increase in the number of devices and applications has led to a rapid increase in data storage and processing requirements. Cloud computing offers many options that can meet the demands in this context. Therefore, Cloud Computing has caused a big change in the way we live and work [1-12].

But we are now at a point where Cloud Computing alone is not enough to provide the necessary services. The increase in the number of connected devices on the Internet of Things (IoT), the interactions and the amount of 
data raises several issues. Two major problems are limitations in terms of network latency and bandwidth. While cloud-based infrastructures give us access to scalable, on-demand computing and processing services that can scale to IoT requirements, these centralized resources can create unacceptable delays and performance problems for devices that have latency-sensitive applications such as health monitoring and emergency response applications.

The term Internet of Things (IoT) is one of the emerging technologies of recent years and is the basis of many trends such as smart cities, smart homes and e-healthcare [13-20]. Now, with IoT, a new era begins with a post-cloud name, where there will be a large amount of data generated by things that are immersed in our daily lives, and many applications will also be implemented on the edge of these devices to consume these data [21-27]. By 2015-2020, 26.3 billion of these nodes will be connected to the Internet [27-38]. This will cause problems with latency and network bandwith.

The problem of network bandwith can cause damage, as IoT devices will produce so much data that existing networks will not be able to send it all to cloud services in time for processing. This can result in a loss of important information for decision making. Data are increasingly produced at the edge of the network, so it would be more efficient to process data at the edge of the network as well. The process of handling data at the edge is now known as «Edge Computing». Cisco has created the term «Fog Computing» which implies the computation performed at gateways, routers, etc. Fog computing is a distributed model that provides cloud services to peripheral devices on the network. In the following sections of this article, several definitions and descriptions of these two terms will be explained.

Edge Computing also has a strong influence on how Machine Learning methods are implemented. Cloud Computing has enabled a revolution in the scalability of artificial intelligence by allowing model training to be conducted in an affordable and sustainable manner. Since model training generally requires a lot of CPU power, it is estimated that training will still be done in the Cloud computing, but the inference (which is the application of a trained algorithm to a real world) will be done at the edge. The large-scale characteristics of these systems and the complexity of the applications suggest that rethinking both the application model and the development process. As can be seen in the name of Distributed Artificial Intelligence (DAI), the advances in both distributed systems and artificial intelligence are the main fields of 
driving. Edge Computing provides an excellent way to use autonomous software or hardware intelligence agents in a distributed manner [39-47]. This comes with a question: How far will artificial intelligence eventually go?

The objective of this paper is to provide an analysis of edge computing, the shift of computing models from centralized to edge computing, and to share the state of the art on edge computing and models of artificial intelligence distribution. This article is carried out with the aim of continuing the doctoral thesis to create a deep tech architecture for intelligent Internet of Things (IoT) systems.

\section{Edge Computing \& Fog Computing}

Cloud computing has played an important role in the last decade to perform massive and complex data computation, taking advantage of virtualized resources and parallel processing with scalable data storage. However, due to the explosive growth of lightweight connected devices that are driving the entire information society into the Internet of Things (IoT) era, the Cloud is facing increasing challenges in supporting lightweight IoT devices, especially for delay sensitive IoT applications.

Data is increasingly produced at the edge of the network; therefore it would be more efficient to process data at the edge of the network as well. But, compared to the rapid development of data processing speed, the network bandwidth has become a problem. As an example, for the delay-sensitive case, a Boeing 787 will generate about 5 Gigabytes of data every second, but the bandwidth between the aircraft and the satellite or the ground base station is not large enough for data transmission. Another example is that an autonomous car will generate one gigabyte of data every second and requires real-time processing for the vehicle to make the right decisions. If all the data has to be sent to the cloud for processing, the response time would be too long. Not to mention that the bandwidth and reliability of the current network would be threatened by its ability to support a large number of vehicles in a single area. In this case, data needs to be processed at the edge for shorter response time, more efficient processing and less network pressure [48-55].

Internet of Things (IoT) refers to the interaction and communication between billions of devices that produce, and exchange data related to real-world 
objects (co-sas). The characteristics of IoT, which include a large-scale network of things, heterogeneity at the level of devices and networks and the large number of data and events generated by these things, will make the development of various applications and services a very complicated task. Internet of Things (IoT) applications need to handle a variety of information from a large number of heterogeneous devices. In relation to this issue, the Edge Computing (EC) paradigm, with the idea of supporting devices with a cloud closer to the edge of the network, appears as an attractive solution. However, adding Edge resources complicates network management, as several devices will face them [56-63].

Another term that is also a step of cloud-based services through which data is produced is «Fog Computing». Cisco defined the concept of «Fog computing» as an extension of cloud computing to harness the full potential of the IoT. The fog is a layer between the edge and the cloud, which extends the cloud closer to the nodes that produce and act on the IoT data. Industrial gateways, routers and other devices with the necessary processing power, storage capabilities and network connection may be fog nodes. By analyzing and pre-processing the data at these nodes, fog computing can minimize latency and reduce bandwidth usage. In addition to Fog Computing, Edge Computing aims at the same objective. However, additional computing power and device storage at the edge are used [64-70].

Fog computing allows for seamless integration of edge and cloud resources. It supports intelligent, decentralized processing of unprecedented data volumes generated by implemented IoT sensors for seamless integration of physical and virtual environments. This could generate many benefits for society, for example, by enabling intelligent healthcare applications. The further development of fog computing could help the IoT to reach its vast potential [71].

\section{Deep Tech}

Deep tech is a new term introduced by Swati Chaturvedi, co-founder and CEO of the investment company Propel (x). Deep tech projects are, in principle, more socially relevant. By its definition, deep tech refers to «companies founded on the basis of scientific discovery or significant engineering 
innovation» and which also seek to make the world a better place. She describes deep tech: «We define deep tech as companies founded on a scientific discovery or significant engineering innovation. This is where they ask: «Not all technology companies are based on these principles. Most technology companies today are based on business model innovation or on the transition from the offline to the online business model using existing technology. Take the example of Uber: Uber is based on the concept of a «shared economy», an innovative business model that allows individuals to share existing resources.

On the other hand, deep tech companies rely on tangible scientific discoveries or engineering innovations. They are trying to solve big problems that really affect the world around them. For example, a new medical device or technique to fight cancer, data analysis to help farmers grow more food, or a clean energy solution to try to reduce human impact on climate change. Continuing Uber's reference, companies with deep technology in the transport business would include autonomous vehicles, flying cars or other similar transformation technologies [72-74].

There are several examples of deep tech technological innovations in the published article [75]. What makes a deep tech technological project different from the usual technological products is that it aims to solve real life problems through scientific discovery or real technological innovation. For example, in the life sciences, "We will not cure cancer by investing in a health monitoring application. We will not grow more food, improve our energy efficiency or perform more efficient surgery by investing in internet companies or mobile applications. To achieve these goals, we will have to invest in real things - in cancer drugs, new energy sources and storage, new medical devices... That is why it is important to invest in deep technologies - because without these technologies, humanity does not move forward [76].

Deep tech projects/companies promise solutions in a wide range of fields, using techniques such as Big Data, artificial intelligence or deep learning, with a more scientific approach than what is normally seen in media-covered technology companies. They are not the digital companies that have grown the most in recent years (like Facebook or Spotify), and they are not based on innovative business models (like Airbnb). Instead, they solve problems through significant scientific or technological developments. 


\section{Distributed Artificial Inteligence}

Machine Learning (ML) is becoming a part of our lives. There are several main reasons for this:

- The increasing capacity of the computers used to build and train ML models.

- More data capture capability throughout the computing environment, often in the form of low-cost sensors integrated into everyday consumer, business and industrial products.

- The development of new algorithms and approaches that improve the accuracy of ML applications.

- The creation of software toolkits that make the creation and training of ML applications much easier and therefore less expensive.

In addition to these four reasons, there are two other often overlooked factors that are equally important in bringing AI (artificial intelligence) into our lives. These factors are not about where AI is built and trained, but where it is implemented and used:

- A reduction in the cost and an increase in the performance of the chips that make IA «on the edge».

- Middleware development allows a wider range of applications to run smoothly.

- on a wider variety of chips.

This leads to a new model of IA that fits what is to come: construction and training, which will continue mainly on increasingly powerful cloud-based computers, and inference that is the implementation of the trained ML model.

Cloud computing has enabled a revolution in AI scalability; supporting ML by allowing model training to be conducted in an affordable and sustainable manner. If you had a large data set and had to study it, you could spin a thousand virtual machines to force your way through it. ML basically consists of two main steps:

Training is when a data set is studied and the results are incorporated into an algorithm of some kind. For example, you must study one million pictures 
of cats to learn how to identify future cat images. This process requires massive data and is computationally intensive.

Inference is the application of a learned algorithm to a real-world problem. Algorithm training is about the past, inference is about the present. Teaching the model to identify a cat is training while taking a single picture and using that algorithm to decide if it is a cat or not is an inference.

Where should the calculations be made to make the inference? The short answer is that for many - if not most - applications, inference in the future will be made at the edge, that is, where the data is collected. This will have a big impact on the way the LMA will be developed. The Edge is the next stage in the evolution of AI technology. The applications that people use in real world products, such as controlling devices in the home or assisting the driver in a car, are all running on the edge and many will require real-time responses. Any delay from bouncing information to the cloud and back could be a problem.

There are many popular research topics related to the ML, including multiagent systems, intelligent transport systems, multi-robot systems, wireless sensor networks and intelligent network systems. The technology behind this is called distributed artificial intelligence (DAI) or distributed intelligence (DI). As the term DAI implies, advances in both distributed systems and artificial intelligence are its main driving forces. There are many ID applications such as Smart Grid, Intelligent Surveillance, Industrial Control and Automation, Wireless Sensor Networks, Multi-Robots, etc. How to train a high accuracy model and how to use the inference model on distributed architecture are two important topics of AI research. At work, there are two ways to do this:

1. Training on distributed architecture

2. Inference about distributed architecture.

This work proposes three layers of architecture: Sensor Layer, Fog Layer, Cloud Layer and Actuator Layer.

\section{Conclusion}

In this article, we discuss the current state of Cloud Computing, Edge \& Fog Computing and its relationship to Machine Learning. We can conclude 
that thanks to IoT and many other developments, most of the calculations will be done at the edge, there is a big movement from centralized cloud services to distributed edge models. As the price of computing continues to fall, the number of computing devices will continue to increase exponentially. While that cannot persist indefinitely, one thing seems certain: computational devices powered by artificial intelligence will touch our lives in almost every conceivable way. The power, safety, and speed requirements of these devices require inference at the edge, where data is collected. This will allow for an increasingly common way of identifying the digital devices that will play a role in our lives.

\section{Future work}

The next phase of this work is to investigate deep tech architectures that focus on an artificial intelligence distribution model and, after analyzing them, to propose and create a deep tech architecture for intelligent IoT systems.

\section{References}

1. Armbrust, M., Fox, A., Griffith, R., Joseph, A. D., Katz, R., Konwinski, A., ... Zaharia, M. (2010). A View of Cloud Computing. Commun. ACM, 53(4), 50-58.

2. Corchado, J., \& Lees, B. (1998). Case based reasoning opportunities and technologies. In CONFERENCE ON KNOWLEDGE DISCOVERY. IEE, SAVOY PLACE, LONDON.

3. Corchado, J., \& Lees, B. (1998). Artificial neural networks in pattern recognition: multicollinearity and heterocedasticity. In COLLOQUIUM ON KNOWLEDGE DISCOVERY. LONDON, UK.

4. Corchado, J., \& Lees, B. (1998). An overview of intelligent frameworks. In COLLOQUIUM ON INTELLIGENT SYSTEMS. IEE, LONDON, UK.

5. Corchado, J. M., \& Lees, B. (1998). Probis: Modelling intelligence with hybrid systems. In WORKSHOP ON DATA MINING. University of GLASGOW, SCOTLAND, UK.

6. Corchado, J. (1998). Real time forecast with intelligent systems. In CONFERENCE ON KNOWLEDGE DISCOVERY. IEE, SAVOY PLACE, LONDON.

7. Corchado, J. M. (1997). Bdi multiagent hybrid architecture for project management. In IEEE COLLOQUIUM ON KNOWLEDGE DISCOVERY AND DATA MINING. LONDON ENGLAND. 
8. Corchado, J. M. (1997). System for decision making: a practical case. In CONFERENCE ON KNOWLEDGE DISCOVERY AND DATA MINING. IEE, LONDON, UK.

9. Corchado, J. (1995). Cbr systems, an overview. In INTERNATIONAL CONFERENCE ON INTELLIGENT SYSTEMS. LONDON, ENGLAND, UK.

10. Li, T., Sun, S., Corchado, J. M., \& Siyau, M. F. (2014, July). A particle dyeing approach for track continuity for the SMC-PHD filter. In 17 th International Conference on Information Fusion (FUSION) (pp. 1-8). IEEE.

11. Corchado, E. S., Corchado, J. M., Sáiz, L., \& Lara, A. (2004, July). A beta-cooperative $\mathrm{cbr}$ system for constructing a business management model. In Industrial Conference on Data Mining (pp. 42-49). Springer, Berlin, Heidelberg.

12. Corchado, J. M., \& Lees, B. (1998). Integration ai models. In WORKSHOP ON KNOWLEDGE DISCOVERY AND DATA MINING. PML-NERC, PLYMOUTHLONDON, UK.

13. Corchado, J. M., \& Lees, B. (1998). Cognitive models for integrating artificial intelligence approaches. In AII WORKSHOP ON KNOWLEDGE DISCOVERY. GLASGOW, UK.

14. Corchado, J. M. (1997). Real time forecast with intelligent systems: Cbrs and anns. In WORKSHOP ON ARTIFICIAL NEURAL NETWORKS. ABERDEEN (pp. 1-3).

15. Casado-Vara, R., Prieto, J., De la Prieta, F., \& Corchado, J. M. (2018). How blockchain improves the supply chain: Case study alimentary supply chain. Procedia computer science, 134, 393-398.

16. Corchado, J. M. (1996). Case-base reasoning recommendation system. In IEEE COLLOQUIUM ON KNOWLEDGE DISCOVERY. LONDON, UK.

17. Corchado, J. M. (1995). Neuro-symbolic reasoning-a solution for complex problemas. In INTERNATIONAL CONFERENCE ON INTELLIGENT SYSTEMS. LONDON, UK.

18. Corchado, J. M. (1995). Multi agent tools: a case study. In IEEE COLLOQUIUM ON KNOWLEDGE DISCOVERY. LONDON ENGLAND, UK.

19. Casado-Vara, R., Prieto-Castrillo, F., \& Corchado, J. M. (2018). A game theory approach for cooperative control to improve data quality and false data detection in WSN. International Journal of Robust and Nonlinear Control, 28(16), 50875102.

20. Corchado, J. M., \& Lees, B. (2001). Adaptation of cases for case based forecasting with neural network support. In Soft computing in case based reasoning (pp. 293-319). Springer, London.

21. Rodriguez, J. M. C. (2000). Neuro-symbolic model for real-time forecasting problems (Doctoral dissertation, University of Paisley). 
22. Casado-Vara, R., Chamoso, P., De la Prieta, F., Prieto, J., \& Corchado, J. M. (2019). Non-linear adaptive closed-loop control system for improved efficiency in IoT-blockchain management. Information Fusion, 49, 227-239.

23. Li, T., Sun, S., Corchado, J. M., \& Siyau, M. F. (2014, July). Random finite setbased Bayesian filters using magnitude-adaptive target birth intensity. In 17 th International Conference on Information Fusion (FUSION) (pp. 1-8). IEEE.

24. Rodríguez, S., Gil, O., De La Prieta, F., Zato, C., Corchado, J. M., Vega, P., \& Francisco, M. (2010, May). People detection and stereoscopic analysis using MAS. In 2010 IEEE 14th International Conference on Intelligent Engineering Systems (pp. 159-164). IEEE.

25. Guillén, J. H., del Rey, A. M., \& Casado-Vara, R. (2019). Security Countermeasures of a SCIRAS Model for Advanced Malware Propagation. IEEE Access, 7 , 135472-135478.

26. Corchado, J. M. (1996). Artificial intelligence models: composed systems as a solution. In IEEE COLLOQUIUM ON KNOWLEDGE DISCOVERY. LONDON ENGLAND, UK.

27. Casado-Vara, R., De la Prieta, F., Rodriguez, S., Prieto, J., \& Corchado, J. M. (2018, June). Cooperative Algorithm to Improve Temperature Control in Recovery Unit of Healthcare Facilities. In International Symposium on Distributed Computing and Artificial Intelligence (pp. 49-62). Springer, Cham.

28. Corchado, J. M. (1995). Hybrid cbr system for real-time temperature forecasting in the ocean. In IEEE COLLOQUIUM ON KNOWLEDGE DISCOVERY. LONDON, UK.

29. Corchado, J. M. (1995). A distributed recommendation system assos. In IEEE COLLOQUIUM ON KNOWLEDGE DISCOVERY. IEE, LONDON, UK.

30. Corchado, J. M. (1995). The use of kernel methods in cbr systems. In INTERNATIONAL CONFERENCE ON INTELLIGENT SYSTEMS. LONDON ENGLAND UK.

31. Corchado, J. M. (1995). Case based reasoning systems: automatic construction. In INTERNATIONAL CONFERENCE ON INTELLIGENT SYSTEMS. LONDON ENGLAND UK.

32. Casado-Vara, R., de la Prieta, F., Prieto, J., \& Corchado, J. M. (2018, November). Blockchain framework for IoT data quality via edge computing. In Proceedings of the 1st Workshop on Blockchain-enabled Networked Sensor Systems (pp. 19-24). ACM.

33. Gil, A. B., De la Prieta, F., \& Rodríguez, S. (2011). Automatic Learning Object Extraction and Classification in Heterogeneous Environments. In Highlights in Practical Applications of Agents and Multiagent Systems (pp. 109-116). Springer, Berlin, Heidelberg. 
34. Rodríguez, S., Tapia, D. I., Sanz, E., Zato, C., de la Prieta, F., \& Gil, O. (2010, July). Cloud computing integrated into service-oriented multi-agent architecture. In International Conference on Information Technology for Balanced Automation Systems (pp. 251-259). Springer, Berlin, Heidelberg.

35. Di Mascio, T., Vittorini, P., Gennari, R., Melonio, A., De La Prieta, F., \& Alrifai, M. (2012, July). The Learners' User Classes in the TERENCE Adaptive Learning System. In 2012 IEEE 12th International Conference on Advanced Learning Technologies (pp. 572-576). IEEE.

36. Casteleiro-Roca, J. L., Pérez, J. A. M., Piñón-Pazos, A. J., Calvo-Rolle, J. L., \& Corchado, E. (2015). Modeling the electromyogram (EMG) of patients undergoing anesthesia during surgery. In 10th international conference on soft computing models in industrial and environmental applications (pp. 273-283). Springer, Cham.

37. Prieto, J., Alonso, A. A., la Rosa, de, R., \& Carrera, A. (2014). Adaptive Framework for Uncertainy Analysis in Electromagnetic Field Measurements. Radiation Protection Dosimetry. doi:10.1093/rpd/ncu260

38. Prieto, J., Bahillo, A., Mazuelas, S., Blas, J., Fernández, P., \& Lorenzo, R. M. (2008). RTS/CTS mechanism with IEEE 802.11 for indoor location. Presented at the NAV08/ILA37, The Navigation Conference \& Exhibition.

39. Prieto, J., Bahillo, A., Mazuelas, S., Fernández, P., Lorenzo, R. M., \& Abril, E. J. (2012a). Self-Calibration of TOA/Distance Relationship for Wireless Localization in Harsh Environments. Presented at the 2012 IEEE International Conference on Communications (ICC 2012).

40. Prieto, J., Bahillo, A., Mazuelas, S., Lorenzo, R. M., Blas, J., \& Fernández, P. (2009a). Adding indoor location capabilities to an IEEE 802.11 WLAN using real-time RTT measurements (pp. 113-119). IEEE Press. Retrieved from http:// dl.acm.org/citation.cfm? $\mathrm{id}=1689059.1689083 \&$ coll $=\mathrm{DL} \& \mathrm{dl}=\mathrm{GUIDE} \& \mathrm{CFID}=$ 647274630\&CFTOKEN=45917612

41. Bullon, Juan, et al. "Manufacturing processes in the textile industry. Expert Systems for fabrics production.» ADCAIJ: Advances in Distributed Computing and Artificial Intelligence Journal 6.4 (2017): 15-23.

42. Souza de Castro, Lucas Fernando, Gleifer Vaz Alves, \& André Pinz Borges. «Using trust degree for agents in order to assign spots in a Smart Parking.» (2017).

43. Moung, Ervin. «A Comparison of the YCBCR Color Space with Gray Scale for Face Recognition for Surveillance Applications.» ADCAIJ: Advances in Distributed Computing and Artificial Intelligence Journal [Online], 6.4 (2017): 25-33.

44. Kethareswaran, V., \& C. Sankar Ram. «An Indian Perspective on the adverse impact of Internet of Things (IoT).» ADCAIJ: Advances in Distributed Computing and Artificial Intelligence Journal 6.4 (2017): 35-40. 
45. Cunha, Rafhael, Cleo Billa, \& Diana Adamatti. «Development of a Graphical Tool to integrate the Prometheus AEOlus methodology and Jason Platform.» ADCAIJ: Advances in Distributed Computing and Artificial Intelligence Journal 6.2 (2017): 57-70.

46. Farias, Giovani Parente, et al. «Predicting Plan Failure by Monitoring Action Sequences and Duration.» ADCAIJ: Advances in Distributed Computing and Artificial Intelligence Journal 6.4 (2017): 55-69.

47. Vera, Jefferson Stewart Espinosa. «Human rights in the ethical protection of youth in social networks-the case of Colombia and Peru.» ADCAIJ: Advances in Distributed Computing and Artificial Intelligence Journal 6.4 (2017): 71-79.

48. Casado-Vara, R., Martin-del Rey, A., Affes, S., Prieto, J., \& Corchado, J. M. (2020). IoT network slicing on virtual layers of homogeneous data for improved algorithm operation in smart buildings. Future Generation Computer Systems, 102, 965-977.

49. Mateen, Abdul, et al. «Secure data access control with perception reasoning.» ADCAIJ: Advances in Distributed Computing and Artificial Intelligence Journal 7.1 (2018): 13-28.

50. Teixeira, Eduardo Porto, Eder Goncalves, \& Diana F. Adamatti. «Ulises: A AgentBased System For Timbre Classification.» ADCAIJ: Advances in Distributed Computing and Artificial Intelligence Journal 7.1 (2018): 29-40.

51. Van Haare Heijmeijer, Alexis, \& Gleifer Vaz Alves. «Development of a Middleware between SUMO simulation tool and JaCaMo framework.» ADCAIJ: Advances in Distributed Computing and Artificial Intelligence Journal 7.2: 5-15.

52. Glaeser, Stefania da Silveira, et al. «Modeling of Circadian Rhythm under influence of Pain: an approach based on Multi-agent Simulation.» ADCAIJ: Advances in Distributed Computing and Artificial Intelligence Journal 7.2 (2018): 17-25.

53. de Melo, Maximilian Jaderson, et al. «Robust and adaptive chatter free formation control of wheeled mobile robots with uncertainties.» ADCAIJ: Advances in Distributed Computing and Artificial Intelligence Journal 7.2 (2018): 27-42.

54. Ribeiro, Catarina, et al. «Customized normalization clustering meth-odology for consumers with heterogeneous characteristics.» ADCAIJ: Advances in Distributed Computing and Artificial Intelligence Journal 7.2 (2018): 53-69.

55. Becerril, Anahiby Anyel. «The value of our personal data in the Big Data and the Internet of all Things Era.» ADCAIJ: Advances in Distributed Computing and Artificial Intelligence Journal 7.2 (2018): 71-80.

56. Garcia, Ana Cristina Bicharra, \& Adriana Santarosa Vivacqua. «ACoPla: a Multiagent Simulator to Study Individual Strategies in Dynamic Situations.» ADCAIJ: Advances in Distributed Computing and Artificial Intelligence Journal 7.2: 81-91. 
57. Casado-Vara, R., Novais, P., Gil, A. B., Prieto, J., \& Corchado, J. M. (2019). Distributed continuous-time fault estimation control for multiple devices in IoT networks. IEEE Access, 7, 11972-11984.

58. Jörg Bremer, Sebastian Lehnhoff. (2017) Decentralized Coalition Formation with Agent-based Combinatorial Heuristics. ADCAIJ: Advances in Distributed Computing and Artificial Intelligence Journal (ISSN: 2255-2863), Salamanca, v. 6, n. 3

59. Rafael Cauê Cardoso, Rafael Heitor Bordini. (2017) A Multi-Agent Extension of a Hierarchical Task Network Planning Formalism. ADCAIJ: Advances in Distributed Computing and Artificial Intelligence Journal (ISSN: 2255-2863), Salamanca, v. 6, n. 2

60. Enyo Gonçalves, Mariela Cortés, Marcos De Oliveira, Nécio Veras, Mário Falcão, Jaelson Castro (2017). An Analysis of Software Agents, Environments and Applications School: Retrospective, Relevance, and Trends. ADCAIJ: Advances in Distributed Computing and Artificial Intelligence Journal (ISSN: 2255-2863), Salamanca, v. 6, n. 2

61. Eduardo Porto Teixeira, Eder M. N. Goncalves, Diana F. Adamatti (2017). Ulises: A Agent-Based System For Timbre Classification. ADCAIJ: Advances in Distributed Computing and Artificial Intelligence Journal (ISSN: 2255-2863), Salamanca, v. 6, n. 2

62. Crespo-Ramos, M. J., Machón-González, I., López-García, H., \& Calvo-Rolle, J. L. (2013). Detection of locally relevant variables using SOM-NG algorithm. Engineering Applications of Artificial Intelligence, 26(8), 1992-2000.

63. Aversa, R., Petrescu, R. V., Akash, B., Bucinell, R., Corchado, J., Berto, F., ... \& Petrescu, F. I. (2017). Kinematics and forces to a new model forging manipulator. American Journal of Applied Sciences, 14(1), 60-80.

64. Li, T., Sun, S., Bolić, M., \& Corchado, J. M. (2016). Algorithm design for parallel implementation of the SMC-PHD filter. Signal Processing, 119, 115-127.

65. Corchado, J. M., Aiken, J., Corchado, E. S., \& Fdez-Riverola, F. (2005). Evaluating the air-sea interactions and fluxes using an instance-based reasoning system. AI Communications, 18(4), 247-256.

66. Costa, Â., Novais, P., Corchado, J. M., \& Neves, J. (2011). Increased performance and better patient attendance in an hospital with the use of smart agendas. Logic Journal of IGPL, 20(4), 689-698.

67. Rodríguez, S., de La Prieta, F., Tapia, D. I., \& Corchado, J. M. (2010, June). Agents and computer vision for processing stereoscopic images. In International Conference on Hybrid Artificial Intelligence Systems (pp. 93-100). Springer, Berlin, Heidelberg. 
68. Corchado, J. M., Corchado, E. S., \& Pellicer, M. A. (2004, September). Design of cooperative agents for mobile devices. In International Conference on Cooperative Design, Visualization and Engineering (pp. 205-212). Springer, Berlin, Heidelberg.

69. Corchado, J. M., Laza, R., Borrajo, L., Yañez, J. C., De Luis, A., \& Gonzalez-Bedia, M. (2003, July). Agent-based web engineering. In International Conference on Web Engineering (pp. 17-25). Springer, Berlin, Heidelberg.

70. Fdez-Riverola, F., \& Corchado, J. M. (2003). Forecasting red tides using an hybrid neuro-symbolic system. AI Communications, 16(4), 221-233.

71. González-Briones, A., Prieto, J., De La Prieta, F., Herrera-Viedma, E., \& Corchado, J. (2018). Energy optimization using a case-based reasoning strategy. Sensors, $18(3), 865$.

72. Díaz, F., Fdez-Riverola, F., Glez-Peña, D., \& Corchado, J. M. (2006, September). Using fuzzy patterns for gene selection and data reduction on microarray data. In International Conference on Intelligent Data Engineering and Automated Learning (pp. 1087-1094). Springer, Berlin, Heidelberg.

73. Koetsier, J., Corchado, E., MacDonald, D., Corchado, J., \& Fyfe, C. (2004, June). Kernel maximum likelihood hebbian learning. In International Conference on Computational Science (pp. 650-653). Springer, Berlin, Heidelberg.

74. Pavón, J., \& Corchado, J. (2004). Agents for the web. International journal of Web engineering and technology, 1(4), 393-396.

75. Fdez-Riverola, F., Díaz, F., Borrajo, M. L., Yáñez, J. C., \& Corchado, J. M. (2005, August). Improving gene selection in microarray data analysis using fuzzy patterns inside a cbr system. In International Conference on Case-Based Reasoning (pp. 191-205). Springer, Berlin, Heidelberg.

76. Fernández-Riverola, F., \& Corchado, J. M. (2003, November). Employing tsk fuzzy models to automate the revision stage of a cbr system. In Conference on Technology Transfer (pp. 302-311). Springer, Berlin, Heidelberg. computing in IoT applications, pp. 41-54 